\title{
The development and application of an optimization tool in industrial design
}

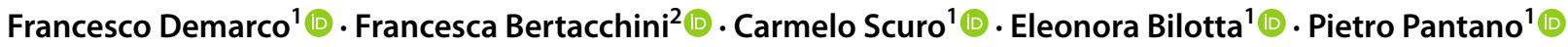

Received: 30 April 2020 / Accepted: 24 July 2020

(c) The Author(s) 2020, corrected publication 2020

\begin{abstract}
Designers are identified, in industry 4.0, as the professional figures that have to design well performing new object. In order to do this, it is necessary to take into account a series of properties called design objectives. Often the structural problem of new design is underestimated or even not considered. This can be a real problem because this objective is in competitive and in contrast with other design objectives. So, this can bring to substantial change in a design in the final phase and sometimes to the complete change. In this paper is presented an optimization workflow that adopt a Multi Objective Optimization engine so called "Octopus" and Karamba3D, that is a Finite Elements (FE) plug-in, typically used in structural simulations, these extensions run in a software: Grassopper3D, that is a parametric design tool. The workflow allows designers to explore a large range of solutions and at the same time permits to filter and sort the optimized models in order to analyze the tradeoff of the resultant solution space, both qualitatively and quantitatively. In such way designers can obtain easily a lot of information of the generate design and identify potential solution for immediate use or for further optimization. In this paper is analyzed a design problem of an ergonomic chair in order to provide the efficiency of the workflow. The design criteria and the structural problem for this type of design object are identified as the main optimization objectives in order to iteratively improve the design solutions.
\end{abstract}

Keywords Parametric design · Genetic algorithms · Optimization tool $\cdot$ Industry 4.0

\section{Introduction}

To date, designers face the challenge of designing products by improving the performance of objects using qualitative and quantitative criteria. Using traditional design methods, the new class of designers are unable to produce and

\author{
Carmelo Scuro \\ carmelo.scuro@unical.it \\ Francesco Demarco \\ francesco.demarco@unical.it \\ Francesca Bertacchini \\ francesca.bertacchini@unical.it \\ Eleonora Bilotta \\ eleonora.bilotta@unical.it \\ Pietro Pantano \\ pietro.pantano@unical.it \\ 1 Department of Physics, University of Calabria, Rende, Italy \\ 2 Department of Mechanical, Energy and Management \\ Engineering, University of Calabria, Rende, Italy
}

examine a large number of variants of the object, and they cannot guarantee the performance criteria implemented in the original design. The new graphical and computational computer technologies have permit to develop design tools that allows to explore and find a larger number of solution in design subspace of optimum results [29]. A creative optimization method is shown in this paper, employing a multi-objective optimization engine called Octopus. This optimization engine is implemented in Grasshopper3d, a parametric modeling tools for Rhinoceros ${ }^{\circledR}$. In Octopus, the designer can filter and sort the solutions in order to compare them and select the elements of the population (set of solutions), to which to apply subsequent optimizations, making the optimization process interactive. Octopus is based on an evolutionary system so called Genetic Algorithm (GA). Some GA techniques allow designers to act, if necessary, in the evolutionary optimization process by selecting which elements of the population to maintain and which to discard $[14,15]$. For this reason, these techniques are defined as IGA (Interactive Genetic Algorithm) [34], while the evolutionary method is called 
Evolutive Multi-objective Optimization (EMO) [56]. Different design criteria are examined, in the production process of the object, as optimization objective. The solutions obtained are analyzed by the designer and are then implemented again by the combined action between the computer and the user. At the end of the process, It is possible to identified as the system is based on two different elements that allow its success: the evolutionary systems GA and user intervention [4, 20, 49]. The limited traditional methods related to the design of objects have led researchers to increasingly adopt the GA especially in the initial stages of the project. To date, the design of objects is a function of ever more complex geometries and shapes. For example, the furnishing element taken into consideration in this paper, the chair, must have a series of characteristics that require important design choices to be made by the user. In this way, human interaction with the genetic algorithm becomes fundamental to ensure comfort and increasingly complex geometries.

When a furniture company start to project a chair the design criteria adopted are several and very often in conflict with each other. These criteria include maximum comfort, maximum resistance to loading, stability of the structure and a minimum consumption of raw material in order to minimize cost of production. In addition to these criteria, the design constrains had to be take into account; these must be seen as initial boundary conditions of the problem [48]. These constrains includes size constrains, that are linked to the segment of product for which the design is intended; and the shape constrains that reflect the creative will of the designer and represent the qualitative and aesthetic control parameter of the shape. The exploration in the design space is limited by these boundary condition but they are also necessary to define a path to develop the optimization of the final product.

In traditional process for digital design, such as automotive design, industrial design, appliance design and architecture the responsibility to develop and satisfy different criteria is divided within different departments. Due to this distribution of responsibility, to each criterion is not given a formal weighting and the interaction between criteria remain unexplored. For these reasons, a real process of design improvement doesn't take place. A long process of negotiation between the various department is necessary to achieve a univocal result and to prevent radical changes caused by the prevalence of one department over the other. The building design problem is the clearest example of this, since the design architectural department is always in contrast with the engineers that design the concrete or steel supporting structure of the building. This is not the only example that it is possible to find in the different fields process of design. In addition, the chair design example, permitted to demonstrates the difficulty in the way of development of qualitative criteria (comfort parameters, shape) and the quantitative ones (resistance to load, stability, cost of production).

This research project describes an optimization workflow which combines an EMO engine (Octopus, a Grasshopper3D extension) with parametric design, ergonomic function, material consumption, static equilibrium and structural analysis (using Karamba3D) to accelerate the design cycle and systematically generate, evaluate and explore design option in faster manner respect to conventional design process. This type of workflow, in contrast with traditional practice, generate optimized 3D model computed parametrically and developed algorithmically [19, 21, 39, 41]. Due to the large number of alternatives produced the solutions are presented by multi-dimensional plot often related to a matrix of semi render design representing the subspace of optimum solutions. To demonstrate the efficiency of the proposed generative framework a complete chair optimization problem is developed in Grasshopper3D. The purpose of the proposed method is to show that concepts as EMO and FEM simulation can be used in the design and construction of furnishing objects, in order to optimize production processes.

The paper is divided in five sections: in the section two the theoretical background is outlines; in the section three the description of the optimization chair problem is provide; in section four the result of the optimization process are discusses and in the last section the conclusions are exposed.

\section{Background}

Parametric design system is a method to generate a design by using an explicit dataflow in the form of a graph. This graph is so called Direct Acyclic Graph (DAG) and represent the geometric constrain and the grammar that describing the 3D model in its all parts [13, 25, 26, 30, 31, 42, 43, 50]. The change of the model can be made by adjusting the input parameters. In a previous work this theme of the implementation of the DAG in order to obtain different forms of a jewelry ring based on geometries coming from the chaos theory was addressed by the authors $[19,21,23,24,25,26,28,30$, $31,36,41]$. Parametric design permits to fully describes a design space that identifies all the possible combination of the object analyzed and obtained by changing the input parameters. In the case of a design project characterized by $\mathrm{N}$ parameters the design space is represent as an $\mathrm{N}$ dimensional space. The DAG, describe the design by associating parameters and functions, and govern how design space be explored when input parameters are adjusted [6]. Each design alternative obtained by this method represent a point on the $\mathrm{N}$-dimensional design space. The manual method of search in parametric space allows to find and evaluate only a small set of solutions. The introduction of computationally intense, heuristic search of design allows to generate and 
then explore a very large number of solutions, and often, permits to discover new and unexpected ideas [32].

\subsection{Multi-objective optimization with genetics algorithms}

Multi-objective design optimization is defined as the problem of finding a vector of decision variables that optimizes a vector function whose elements represent multi objective function. The basis of multi objective optimization derive from the need to achieve compromise decision-making in a problem of many conflicting objectives. The aim of the optimum search is to explore a suboptimal solutions space, where each solution presents a different level of objective satisfaction and not in order to find a singular optimum solution.

The use of Evolutionary Computation (EC) is now a wellestablished technique in multi-objective optimization problem. A multi-objective optimization problem implemented whit EC is so called Evolutionary Multi-Objective Optimization (EMO) [12]. In particular, a sub set of EC called Genetic Algorithms (GA), a method inspired to biological mechanism, has found large use in the problems of design optimization [17, 18, 35]. In Fig. 1is shown an example of GA flow chat.

\subsection{Multi-objective optimization in parametric design}

The application of EMO techniques in design found its first experimentation in architecture [37] and, later, found widespread use thanks to the integrated employment with parametric modeling, that in the time becoming a discipline identified as performance-based design [44]. Subsequently, evolutionary optimization techniques were also applied to industrial design problem. In the present work, the previous described techniques are applied at the creation and the optimization of a parametric chair [33]. The design theme of the chair is not a generic design problem, it is in fact one of the themes most addressed by the designers of contemporary history with a very great field of aesthetic and ergonomic solutions. Chair design topic was also one of the main problem faced by the early automatic 3D modeling system, in particular Bentley, in his work on evolutionary design [11, 12], used "clipped stretched cuboids" to create the representation of design objects such as coffee tables [11]. However, the shape is the starting point and should be consider as sketches of ideas rather than finished design. Recently there has been considerable progress in computer graphics [27, 40, 57], September) [14, 15], August) [16, 22], this allows even more complex shape to be generated as shown in the work of Reed and Gillies [52] that creates generative system capable of producing a vast range of semi-realistic

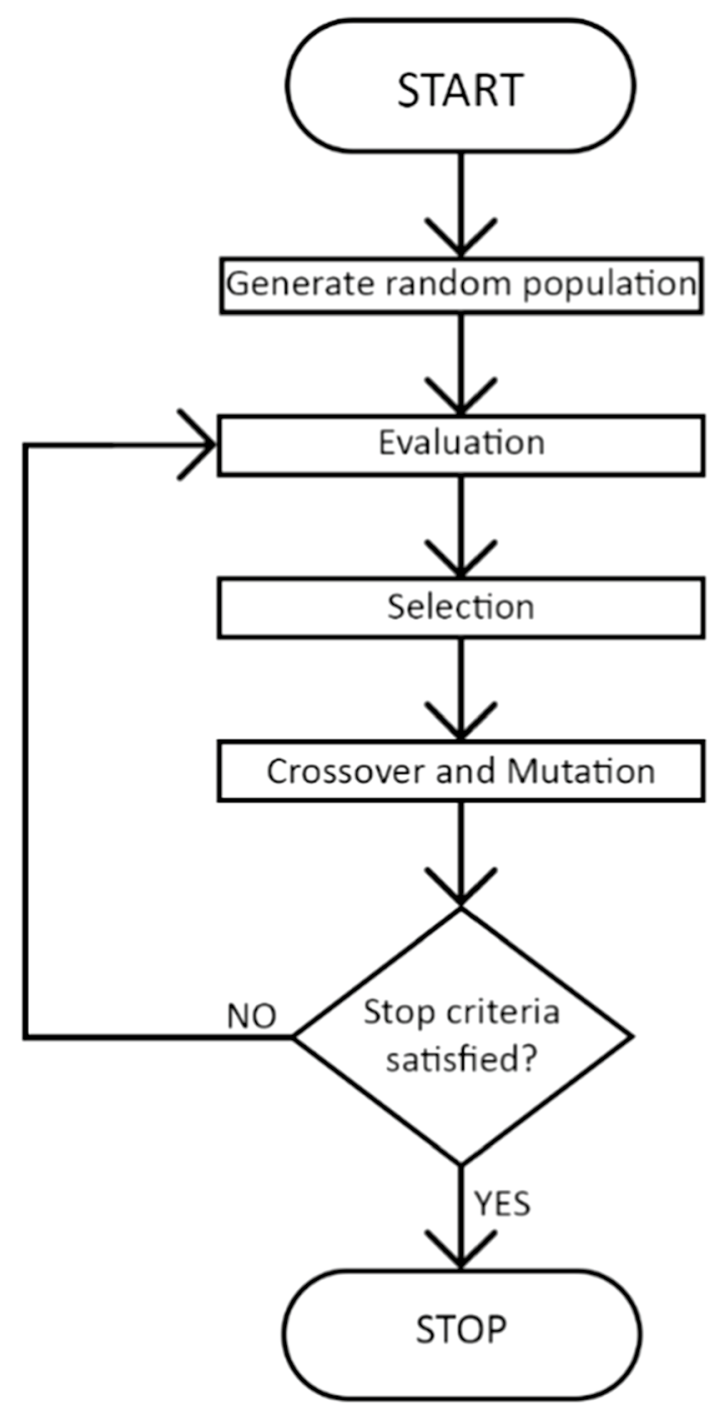

Fig. 1 Genetic algorithm flow chart

3D models. In the specific, the design optimization tools, such as those used for the coffee table, are creation tools that use parametric modeling, performance simulation and mathematical optimization in order to systematically generate and evaluate design alternatives [46]. Another important work to be taken into account is by Kolarevic, in his book he developed a Performance-driven optimization tool that produces a range of solutions in the design space based on multiple performance targets [47]. The solutions obtained through a qualitative and quantitative feedback system were assessed, making this work the precursor of the optimization tools commonly used today.

\subsection{Octopus: multi-objective optimization engine}

This paper is based on the development of a workflow of an optimization tools consisting in the integration of three 
components: parametric design, simulation tools and genetic algorithm [16, 22, 38]. The fulcrum of this workflow is the optimization engine Octopus [58] capable to applying the evolutionary algorithm to any parametric model created in Grasshopper3D.

Pareto-based optimization Techniques are currently the most used techniques. The solutions presented at the end of the optimization process, Pareto Front, are mathematically equivalent, and are characterized by high levels of satisfaction of the objectives. Therefore, all well-performing solutions allow designers to introduce subjective parameters to ensure correct choice of the solution.

Octopus uses Strength Pareto Evolutionary Algorithm (SPEA-2) [61] in combination with Hypervolume Estimation Algorithm (HypE), in order to achieve multi objective optimization [10]. The GA engine start an iterative process that generates individuals (solutions), whose set constitutes a population, at time $\mathrm{t}$, the individuals constituting the population will be the parents of the next generation solutions identified at time $t+1$. This method, used in order to solving optimization problem, emulate the biological evolution and adopt a "fitness function" to coordinate the objective and improve the fitness of solutions, generation after generation. Elitism, mutation rate, mutation probability, crossover rate, population size and maximum generation are the main parameters that can be fixed by the designer, manually. The parameters listed above, are necessary to identify the set of non-dominated solution that constitute the Pareto optimal Set [59]

Multi objective optimization (MOO) allows to solve problems that present multiple objectives to be optimized even if conflicting with each other, enabling to find mediated solutions of these [8]. Although MOO has this important advantage, allowing to take into consideration from the early design stages the parameters that relate to the feasibility and performance of the final object, it is not frequently used, especially in the creation of industrial design objects. There are several reasons which is why this technique is not widespread. The designers must manually identify the solutions in the Pareto optimal set in order to evaluate the compromises of modeling and this can involve to analyzing a large amount of solutions. In addition, a high computational burden is required to analyze and manage the information of the optimization process. In literature, in the architectural sector, a workflow has been introduced. It allows to manage, represent, sort and filter the large amount of data obtained at the end of the optimization process in a more effective way [8].

\section{Methods}

The main purpose of this paper is to apply well-established techniques, used in the field of large-scale design, such as the optimization tools, in the industrial design sector. This allows to demonstrate the great advantages of using these methods in the development of projects in different sectors. In particular, in the industrial design, where the performance design of models, which is the behavior of an object subjected to operating loads, is overlooked. Often, neglecting these characteristics, involves important changes to be made at the end of the process. To demonstrate the veracity of this methodology, a complete example of Multi Objective Optimization of a Chair was developed. As in the classic optimization tools, also in this application, three elements were combined: parametric modeling, simulation of the behavior under operating loads, genetic algorithm. For the realization of this complete example, existing plug-ins for Rhinoceros have been used: Grasshopper3D for parametric modeling, Octopus for MOO and Karamba3D for structural analysis [51] (Fig. 2).

Karamba3d is a structural analysis plug-in for Grasshopper 3D that allows to combine the parametric design environment whit structural analysis. The plug-in takes the parametrized $3 \mathrm{~d}$ model and model it into beams and shell elements, allowing to associate different sections and joints.

In the structural program, it is possible to define force systems, representative of the loads, and constraint conditions that limit the displacements of the structure. This allows the conversion of the parameterized object into a parameterized structure through this definition system. The structural frame of the chair, which constitutes the seat load transmission system, is constituted by the use of slender beams while the seat was modelled by the use of shell elements. Specifically, the slender beams adopt circular hollow aluminum sections. The thickness of the section is characterized by two parameters that represent the objective optimization of the displacement of the structure under load and the relative consumption of raw material during the realization of the object. The shell element characterized by a wooden section, remained constant in the single numerical analysis, and ranged through a numerical slider throughout the calculation process. The material adopted for the modeling of the seat has been assumed orthotropic with the layers orthogonal to the load; the characteristic values of the wood used for the seat are shown in Table 1.

\subsection{Fem introduction}

The FEM analysis were conducted with the plugin Karamba3D, using an optimization approach at the numerical problem. The chair model was schematized using two different structural elements, the beam model for the load-bearing structure of the chair and the shell element for the seat. In addition, the object was modeled by assigning two materials, wood to the seat and aluminum to the structure. The use of different materials led to the implementation of two different perfectly plastic elastic constitutive laws, characterized 


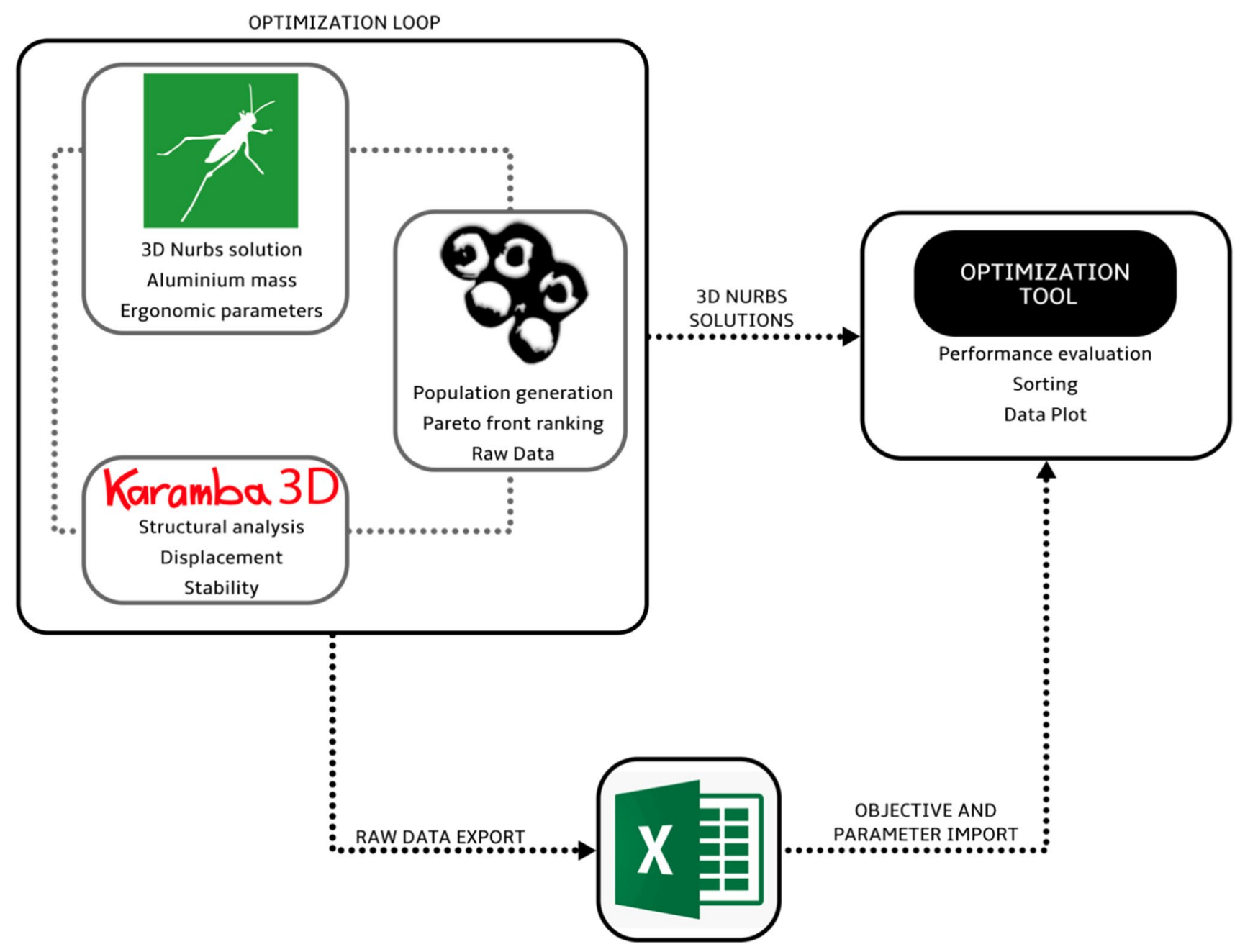

Fig. 2 Adopted workflow

Table 1 Value used for wood's orthotropic behavior definition

\begin{tabular}{ll}
\hline Propriety & Value (units) \\
\hline E1 & $1100(\mathrm{kN} / \mathrm{cm} 2)$ \\
E2 & $37(\mathrm{kN} / \mathrm{cm} 2)$ \\
G12 & $69(\mathrm{kN} / \mathrm{cm} 2)$ \\
G31 & $69(\mathrm{kN} / \mathrm{cm} 2)$ \\
G32 & $50(\mathrm{kN} / \mathrm{cm} 2)$ \\
Gamma & $4(\mathrm{kN} / \mathrm{m} 3)$ \\
Fy1 & $3.6(\mathrm{kN} / \mathrm{cm} 2)$ \\
Fy2 & $2.4(\mathrm{kN} / \mathrm{cm} 2)$ \\
\hline
\end{tabular}

by variation of the engineering constants of the individual materials as shown in Table 1 . E is the elastic modulus, $G_{12}$ the tangential elastic modulus in the $\mathrm{x}-\mathrm{y}$ plane, $\mathrm{G}_{3}$ tangential elastic modulus along the $\mathrm{z}$ direction, $\mathrm{f}_{\mathrm{y}}$ is the tensile yield strength and $\gamma$ the specific weight. In addition, it is useful to highlight how two different behaviors have been assigned to the materials: isotropic for aluminum and orthotropic for wood. This is due to the conformation of the different materials; aluminum has the same behavior in all directions, while wood, due to its conformation with overlapping layers, exhibits different behaviors along the orthogonal directions. As prescribed by the technical standard [3] the analysis was carried out by applying the load in two different steps. in the first the action of the force of gravity and the relative weight of the structural components was applied to the chair, and in the second step, the total weight of a person's body that the object should support was assigned, calculating the deformation and the stress state of the elements. The technical standard restrictions that the analysis must be conducted with a total weight of $110 \mathrm{~kg}$ and that it is distributed evenly according to the human body on the seat and on the parts in contact in $\mathrm{N} / \mathrm{mm}^{2}$. In addition, every single element at the end of the analysis must be in operation in the elastic phase without ever plasticizing. This implies that the strain that the chair has during the loading phase is reversible and that it can return to its initial conformation. In order to be in a 
state of greater design security, the analysis was carried out by applying a weight of $150 \mathrm{~kg}$.

Parametric analysis was carried out by varying 10 parameters over time: seat depth, seat width, armrest height, armrest rotation, backrest height, backrest rotation, rounding edge, structural cross section diameter, cross section thickness and seat thickness. The structural elements of the chair were modelled using a circular hollow beam element characterized by a control parameter for variation of the section thickness. The Timoshenko beams model is implemented and allow for transverse shear deformation. They can be used for thick as well as slender beams [53]. For beams made from uniform material, such the case of the structure of the chair, shear flexible beam theory can provide useful results for cross-sectional dimensions up to $1 / 8$ of typical axial distances. Beyond this ratio the approximations that allow the member's behavior to be described solely as a function of axial position no longer provide adequate accuracy. The Timoshenko beam elements use cubic interpolation functions, which makes them reasonably accurate for cases involving distributed loading along the beam.

The moments of inertia with respect to the centroid are defined as [53]

$$
\begin{aligned}
& I_{11}=\int_{A}\left(x_{2}-x_{2}^{C}\right)^{2} d A \\
& I_{22}=\int_{A}\left(x_{1}-x_{1}^{C}\right)^{2} d A \\
& I_{21}=\int_{A}\left(x_{2}-x_{2}^{C}\right)\left(x_{1}-x_{1}^{C}\right) d A
\end{aligned}
$$

where $\mathrm{x}_{1}$ and $\mathrm{x}_{2}$ are the position of the point in the local beam section axis system and $\mathrm{x}_{1}{ }^{\mathrm{C}}$ and $\mathrm{x}_{2}{ }^{\mathrm{c}}$ are the position of the centroid of the cross-sectional area.

Bending stiffness and rotary inertia contributions for a meshed section profile are calculated using the two-dimensional cross-section model. The following integrated properties are defined for the entire cross-section model meshed with warping elements:

$$
\begin{aligned}
& (E I)_{11}=\int_{A} E\left(x_{2}-x_{2}^{C}\right)^{2} d A \\
& (E I)_{22}=\int_{A} E\left(x_{1}-x_{1}^{C}\right)^{2} d A \\
& (E I)_{21}=\int_{A} E\left(x_{2}-x_{2}^{C}\right)\left(x_{1}-x_{1}^{C}\right) d A \\
& (\gamma I)_{11}=\int_{A} \gamma\left(x_{2}-x_{2}^{m}\right)^{2} d A
\end{aligned}
$$

$(\gamma I)_{22}=\int_{A} \gamma\left(x_{1}-x_{1}^{m}\right)^{2} d A$

$(\gamma I)_{21}=\int_{A} \gamma\left(x_{2}-x_{2}^{m}\right)\left(x_{1}-x_{1}^{m}\right) d A$

where $\left(\mathrm{x}_{1}{ }^{\mathrm{m}}, \mathrm{x}_{2}{ }^{\mathrm{m}}\right)$ is the center of mass of the cross section, $\mathrm{E}$ is the Elastic modulus and $\gamma$ is the specific weight of the material.

For closed thin-walled sections, the torsional constant is calculated by the following equation:

$J=\frac{4 A_{c}^{2}}{\oint \frac{1}{t} d s}$

where $\mathrm{t}$ is the thickness of the section, a variable parameter, $A_{C}$ is the area enclosed by the median line of the section, and $\mathrm{s}$ is the length of the median line, measured along the circumference of the section in a counterclockwise direction.

The effective transverse shear stiffness of the section of a shear flexible beam is defined with the following equation:

$\overline{K_{\propto 3}}=f_{p}^{\propto} K_{\propto 3}$

where $\overline{K_{\alpha 3}}$ is the section shear stiffness in the $\alpha$-direction, $f_{p}^{\propto}$ is a dimensionless factor used to prevent the shear stiffness from becoming too large in slender beam elements, $K_{\alpha 3}$ is the actual shear stiffness of the section; and $\alpha=1,2$ are the local directions of the cross-section, this element his characterized by force units.

Linear section behavior of the element beam based on Timoshenko approach, is characterized by axial force, $\mathrm{N}$; bending moments, M1 and M2 about the 1 and 2 beam section local axes; torque, $\mathrm{T}$; and bi-moment, $\mathrm{W}$, that are defined in terms of the axial stress and the shear stress with the Eqs. (12-16) without consider the contribute of temperature variation [60].

$N=E\left(A \varepsilon_{c}+\Gamma_{0} \chi\right)$

$M_{1}=E\left(I_{11} k_{1}-I_{12} k_{2}\right)$

$M_{2}=E\left(I_{22} k_{2}-I_{12} k_{1}\right)$

$T=G J \psi+G I_{p} \omega_{p}$

$W=E\left(\Gamma_{0} \varepsilon_{c}+\Gamma_{W} \chi\right)$

where: $A$ is the area of the section; $I_{11}$ is the moment of inertia for bending about the 1 -axis of the section; $I_{12}$ is the moment of inertia for cross-bending; $\mathrm{I}_{22}$ is the moment of inertia for bending about the 2-axis of the section; $\mathrm{J}$ is 
the torsional constant calculated with $10 ; \Gamma_{0}$ is the sectorial moment of the section; $\Gamma_{W}$ is the warping constant of the section; $\varepsilon_{c}$ is the axial strain measured at the centroid of the section; $\mathrm{k} 1$ is the curvature change about the first beam section local axis; $\mathrm{k} 2$ is the curvature change about the second beam section local axis; $\psi$ is the twist; $\chi$ s the bi-curvature defining the axial strain in the section due to the twist of the beam and $\omega_{p}$ is the difference between the unconstrained warping amplitude, $\omega_{f}$, and the actual warping amplitude, $\omega$. In this case the section are closed thin-walled and $\Gamma_{0}, \Gamma_{W}$, $\chi$ and $\omega_{p}$ are equal to zero.

In order to create the finite element model of the seat, another element was employed, the shell $[54,55]$. These elements are used to model structures in which one dimension, the thickness, is significantly smaller than the other dimensions. Conventional shell elements use this condition to discretize a body by defining the geometry at a reference surface. In this case, the thickness is defined through the section property definition [7]. Conventional shell elements are characterized by displacement and rotational degrees of freedom. The "upper" surface of a conventional shell element is the surface in the positive normal direction and is referred to as the positive face for contact definition. The "bottom" surface is in the negative direction along the normal and is referred to as the negative face for contact definition. The positive normal direction defines the convention for pressure load application and output of quantities that vary through the thickness of the shell [54, 55]. A positive pressure load applied to a shell element produces a load that acts in the direction of the positive normal. In the shell element used that are valid for thick shell problems or that enforce the Kirchhoff constraint numerically and in the finite-strain shell elements it was computes the transverse shear stiffness by matching the shear response for the case of the shell bending about one axis, using a parabolic variation of transverse shear stress in each layer [7]. The triangular shell implemented in order to discretize the orthotropic element was characterized by six degree of freedom per node, a constant strain state for each layer and is not added in-plane rotational stiffness.

The transverse shear stiffness of the section of a shear flexible shell element is definite as:

$\overline{K_{\alpha \beta}^{t s}}=f_{p} K_{\alpha \beta}^{t s}$

where $\overline{K_{\alpha \beta}^{t s}}$ are the components of the section shear stiffness ( $\alpha, \beta=1,2$ refer to the default surface directions on the shell, as defined in "Conventions," or to the local directions associated with the shell section definition); $f_{p}$ is a dimensionless factor that is used to prevent the shear stiffness from becoming too large in thin shells; $K_{\alpha \beta}^{t s}$ is the actual shear stiffness of the section and is defined by the user.

$$
f_{p}=\frac{1}{1+0.25 \times 10^{-4} \frac{A}{t^{2}}}
$$

where $\mathrm{A}$ is the area of the element and $\mathrm{t}$ is the thickness of the shell. When a general shell section definition not associated with one or more material definitions is used to define the shell section stiffness, the thickness of the shell, $t$, is estimated as:

$t=\sqrt{12 \frac{D_{44}+D_{55}+D_{66}}{D_{11}+D_{22}+D_{33}}}$

This parameter $t$ was varied during the numerical analyzes in combination with the thickness of the circular hollow section used to create the structure of the chair. It is useful to underline how laminated plates and sandwich constructions the $K_{\alpha \beta}^{t s}$ are estimated by matching the elastic strain energy associated with shear deformation of the shell section with that based on piecewise quadratic variation of the transverse shear stress across the section, under conditions of bending about one axis (Fig. 3).

\subsection{Definition of constraints and loads}

After the step in which all the parameters relating to geometry are correctly set, for the definition of the structural problem, the constraint conditions and the operating loads must be defined. The user must do the setting of these boundary conditions manually; more accurate is the mathematical representation of these conditions, more effective the simulation will be. For the chair object, it was chosen to impose interlocking constraints on the base; this condition however does not allow taking into consideration the static equilibrium of the structure. In order to prevent possible overturning problems, the minimization of the distance between the center of gravity in the deformed and not deformed configuration of the chair has been introduced as an optimization objective.

For the correct design of the chair, for identifying the loads of use, the structural and geometric constraints, it was decided to refer to recognized regulatory and performance standards, called UNI standard (Italian National Unification authority). Specifically, the adopted standard is also recognized at European level by the CEN (European Committee for Standardization). The performance standards for the seats are identified by UNI EN 1022: 2018 and more specifically by UNI EN 1335 which in its three sections, listed below, defines the principles of ergonomics and the minimum functional requirements

-1335-1 Size determination [1]

-1335-2 Safety requirements [2] 


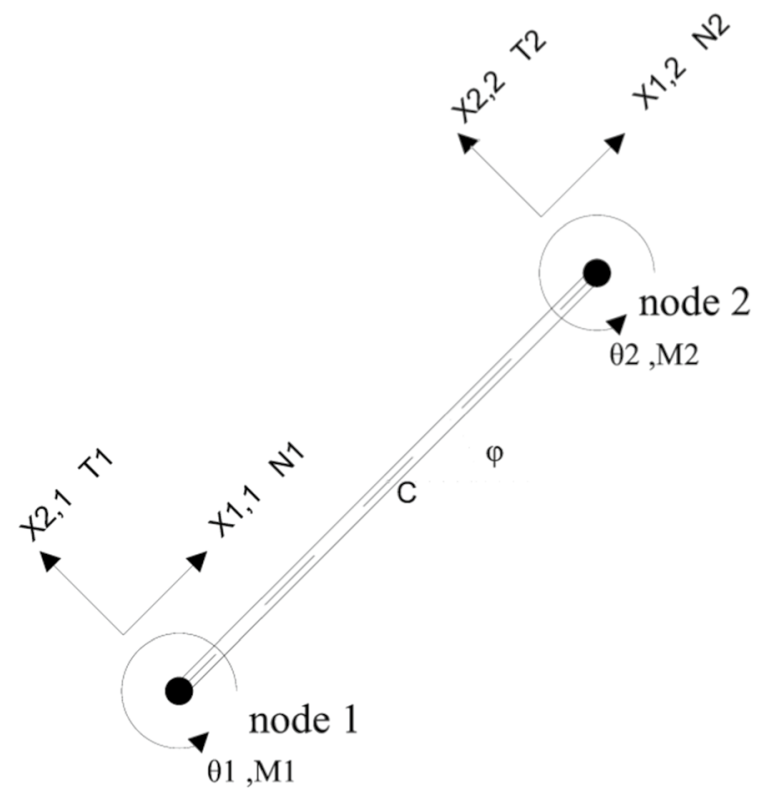

(a)

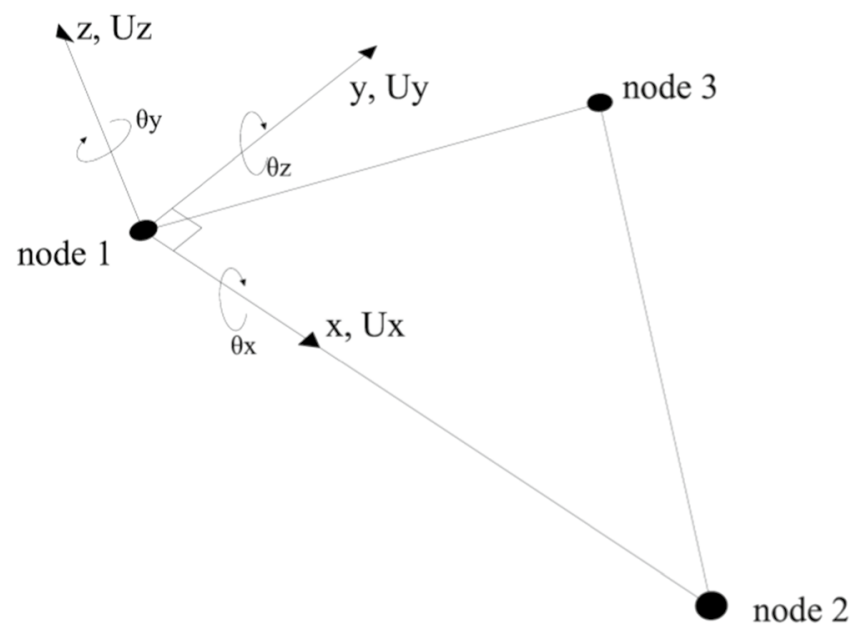

(b)

Fig. 3 a Beam element in its local system. N T M are Normal stress, Shear stress and Bending Moment. C is the centroid. b Flat Triangular shell element, characterized by constant thickness in its local axis system

-1335-3 Safety testing methods [3]

\subsection{Optimization Loop}

For this application, SPEA-2 is use for the multi-objective optimization in Octopus, because the HypE algorithm requires a high computational burden. In fact, the structural simulation tool necessitates to an extraordinary computing power that cannot therefore be used for the genetic algorithm.

The three-dimensional model is optimized in accordance with the different fitness functions that Octopus minimizes. The software allows the User to find the best trade-off between several goals.

For the approach to the application of the "chair" object, the following objectives have been introduced:

- Maximum displacement $\delta$ in $\mathrm{z}$ direction of the chair

- Mass of aluminum used for the structure to reduce the cost of the chair

- Ergonomic Parameters

- Distance between the projection of the center of gravity in the initial and deformed condition (to ensure static equilibrium)

There are 4 objectives and the first two are in contrast to each other. The optimization problem is based on the research of minimum of an unknown function, so, the optimization process, returns as a solution a m-dimensional surface called "fitness landscape". Where, $\mathrm{m}$ is the number of objectives and the fitness landscape, in this case, is represented by a 4th-dimensional surface.

The parametric chair is represented by 10 independent parameters adopted as genes for the optimization problem. The genes selected as parameters of the problem are: seat depth, seat width, armrest height, armrest rotation, backrest height, backrest rotation, rounding edge, structural cross section diameter, cross section thickness and seat thickness. The space of genes is called Genotype, while the space of coded solutions is called Phenotype.

From these last two definitions of the multi-dimensional spaces, it is clear that both the parameter space and the objectives space contain a set of numerical values for each possible alternative. In these spaces, it is possible to represent a solution as a point in an n-dimensional space, where with $\mathrm{n}$ it is possible to indicate respectively the number of parameters or the number of objectives [59]. The spaces of parameters and objectives can be connected in extremely complex ways. For this reason, the greatest effort of search and optimization algorithms will be to overcome the relationships imposed by algorithmic logic that link the spaces of parameters and objectives [9].

As described in paragraph 2, the optimization procedure is constituted by loops. The loop begins with the parameters that form a geometry and a structural model, the algorithm evaluates the solutions of all generations and each of them assigned the level of satisfaction linked to each objective, the fitness value. Before starting the next loop, the solutions are 


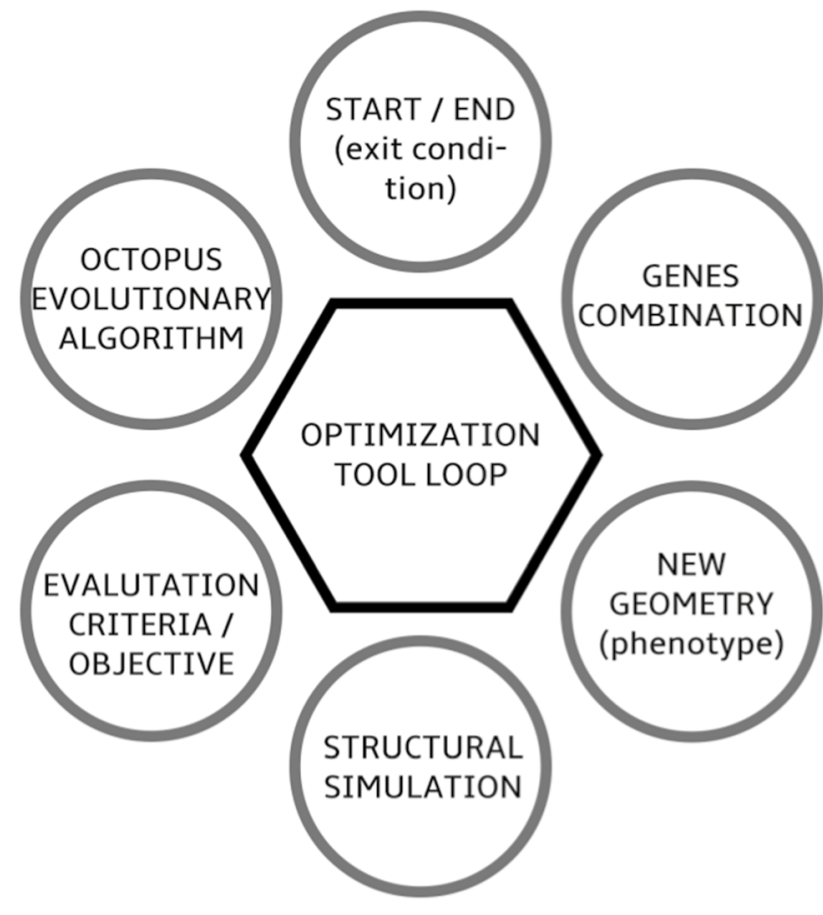

Fig. 4 Optimization loop

Table 2 Parameters used for multi-objective optimization

\begin{tabular}{ll}
\hline Parameter & Value \\
\hline Elistism & 0.5 \\
Mutation probability & 0.1 \\
Mutation rate & 0.5 \\
Cross over rate & 0.8 \\
Population & 100 \\
Initial boost & 2 \\
\hline
\end{tabular}

ranked, and the best of them are crossed in order to generate even better solutions. The described look is showed in Fig. 4 .

This process is regulated by the parameters that can be set by the user. The elitism setting means how much of the non-dominated solutions are stored into the secondary population. Mutation probability controls the chance that an individual mutates. The cross-over rate determines how much of the genes from the parent solutions is combined. A cross-over rate equal to 0.5 means that half of the genes come from either parent. Finally, the size of the population can be chosen. All the values of the parameters, adopted for the multi-objective optimization in Octopus, are shown in Table 2.

The values used are in part default values provided by the software, the user's choices have been related to those that refer to the population and the initial boost. The decision to adopt these values, as suggested by the sector literature, highlighted how it avoids local minimum problems [44, 45].

\section{Results}

For the case study an optimization run was conducted to test the effectiveness of the method. Four objectives were chosen to optimize the problem: Maximum displacement, mass of aluminum used, ergonomic parameters and static equilibrium of the object.

The Octopus optimization engine, described in the previous paragraphs, uses different input data. The first group is represented by the numerical sliders that make up the genes of the parameterized model; the second group of data is represented by the objectives which are numerical values representative of the fittness value of each solution. This data group is always accompanied by a textual datum which describes the objectives and identifies the axes of the solution space. The last input is optional and consists of a mesh representative of the phenotype. This last data is necessary to display the geometry obtained in the solution space. However, the representation of the solution presents a great disadvantage because complex meshes cause an excessive computational burden of calculation and risk ending the analysis before it ends [5]. in this light, optimization techniques are often used in the initial phase of the design process when the models are still sketchy and simple. The case study is characterized by an advanced definition level linked to a static behavior simulation component; it was not possible to use the data relating to the phenotype which was subtracted from the numerical analysis. By eliminating the use of the phenotype data, it was possible to complete the analysis and obtain the Pareto Optimal Set. This present a great disadvantage, in fact, the solutions obtained are represented by cubes and it is not possible to directly display and compare the different individuals obtained. From this process, however, a series of raw data representative of the values assumed by the genes (number slider) and by the objectives were obtained. It is possible to manipulate and sort this data in Excel and re-import it into grasshopper through a plug in called lunchbox. Through these data it is possible to view, compare and select the different individuals, as well as view their performance, which identify, the levels of satisfaction of the different objectives. In this way, the user can view and evaluate each individual simultaneously, quantitatively and qualitatively. the object is shown simultaneously in its geometric shape and its performance, that are displayed through radar chart or parallel coordinate plot chart.

Two distinct.gh files will be generated: the first file compiled in the grasshopper environment, represented in Fig. 5, is the fulcrum of the first phase of the workflow, where, the three-dimensional modeling of the chair including the creation of geometric constraints $(1,2,3)$, the semirealistic representation component (4), the introduction 
of the simulation tool (7), the definition of optimization objectives $(5,6,7)$ are contained and modeled. All these elements then converge in the octopus optimization engine (8).

Raw data are obtained by two distinct methods. They are exported both from the plug in Octopus and from the insertion of data recorders. The latter have the function of storing both the values of the parameters, which generate individuals in subsequent generations, and the values assumed by each target for the specific individual
The second.gh file shown in Fig. 6 was created in order to import the data obtained from the optimization process, using the plug-in lunchbox. Before that, it was necessary to operate on the raw data which are exported to text documents on excel in order to obtain ordered and usable data. After this first phase, the data are analyzed by the functional group (1), The number sliders making up the genes are replaced from new sliders necessary to select generation and individuals, that will correspond to a three-dimensional geometry, a structural model and numerical values representative of the fitness function. The functional groups $(2,3)$
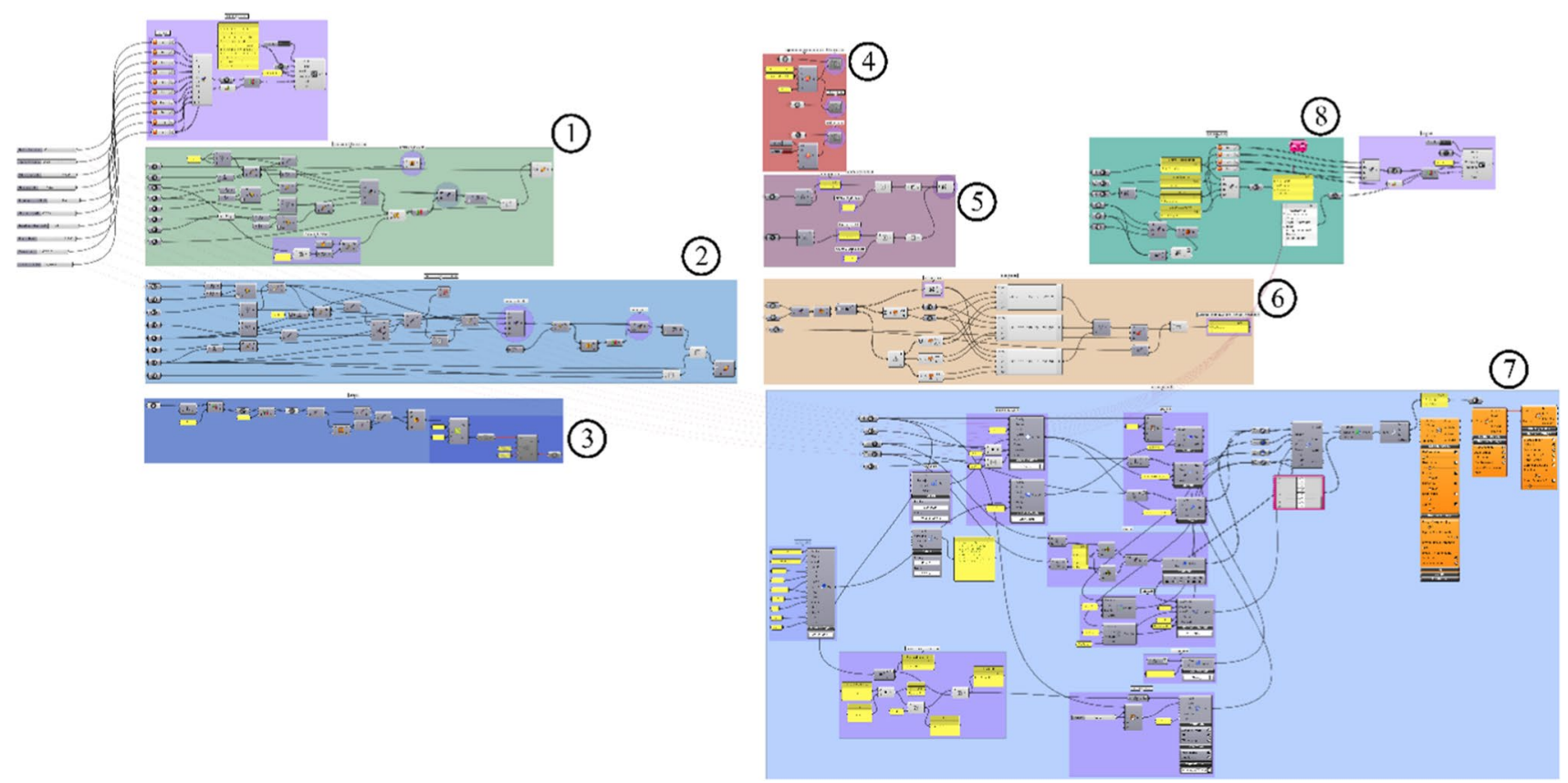

Fig. 5 First gh file

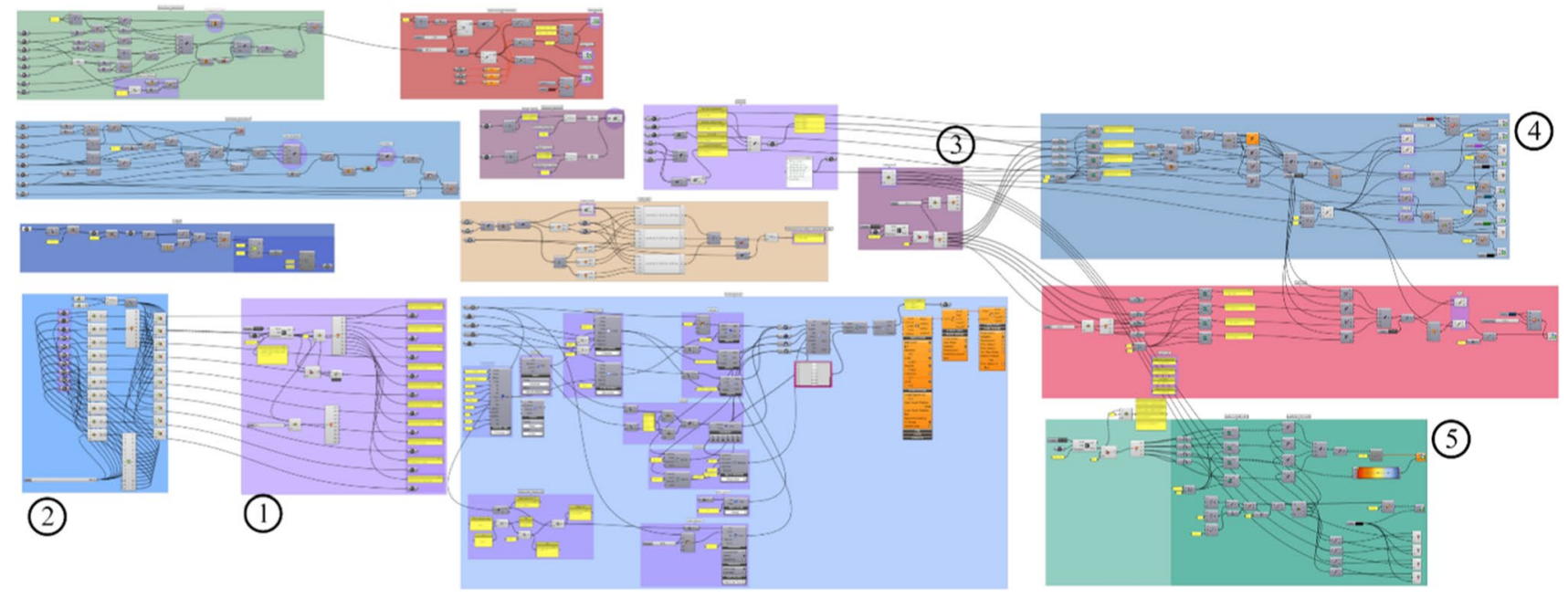

Fig. 6 Second.gh file 
allow to sort the solution respect to global fitness value or respect to a single objective. Groups $(4,5)$ generate the diagrams as parallel plot chart and radar chart.

In the first.gh file, optimization is performed using the parameters introduced inTable 2. At the end of the process, the Pareto Optimal set is obtained, as specified several times. Figure 7 shows the Pareto Front solutions which are those represented by the Delanuy front mesh (1). The other solutions, shown in Fig. 7 (1) are those of previous generations. The transparent color indicates the solution temporally farther compared to those of the Pareto Optimal set. In point 2 of the figure, the Parameter distance graph are represented by coupling the genetic variation of the solution to a polyline. While in point (3) the convergence diagrams are shown, one for each of the $n$ objectives. These diagrams represent the trend of the values assumed by each target over time in a domain scaled between 0 and 1 . The domain of elite solutions is represented in light gray while the Pareto front domain is represented in dark gray.

Octopus plots, in real time, on a three-dimensional graph the optimization results using color and size scales in order to represent the geometric dimensions higher than the third (Fig. 7) Therefore, the software projects points with multidimensional coordinates in three-dimensional space. Each solution is represented by a cube which corresponds only to the mathematical representation of its performance. In light of this, the Pareto optimal set is difficult for the user to understand since it is not possible to visually compare the different designs. Furthermore, the performance of the solutions, characterized by objectives of an order greater than 3 , is difficult to evaluate. Precisely for these reasons it was necessary to define a method that makes it possible to make the data obtained from the analysis easy to comprehend. This last component is show in Fig. 6.

This process allows to obtain, for each individual, structured information on different layers (Fig. 8). it is possible to have the rendering of the geometric model (i) (Fig. 8a) and also a structural model (ii) (Fig. 8b), consisting of beam elements for the frame and shell for the seat, and the related data to the fitness function (iii). Using these data, the corresponding solutions and performances are observed and compared directly.

The run of the optimization was conducted with the hypothesis of minimize the deformation of the chair under load while maintaining at minimum the consumption of raw material and respecting the requirements of the UNI 1335 standard of and In total 10 genes or design variable were manipulated and a total of 5000 design solutions were generated whit an average run time of about $4.5 \mathrm{~s}$ for solution, time required for the computational burden.

In Fig. 9a-c it is possible to observe the two distinct approaches for visualizing the performance of each individual. In order to build these figures, to compare the performances for heterogeneous data, a new existence domain

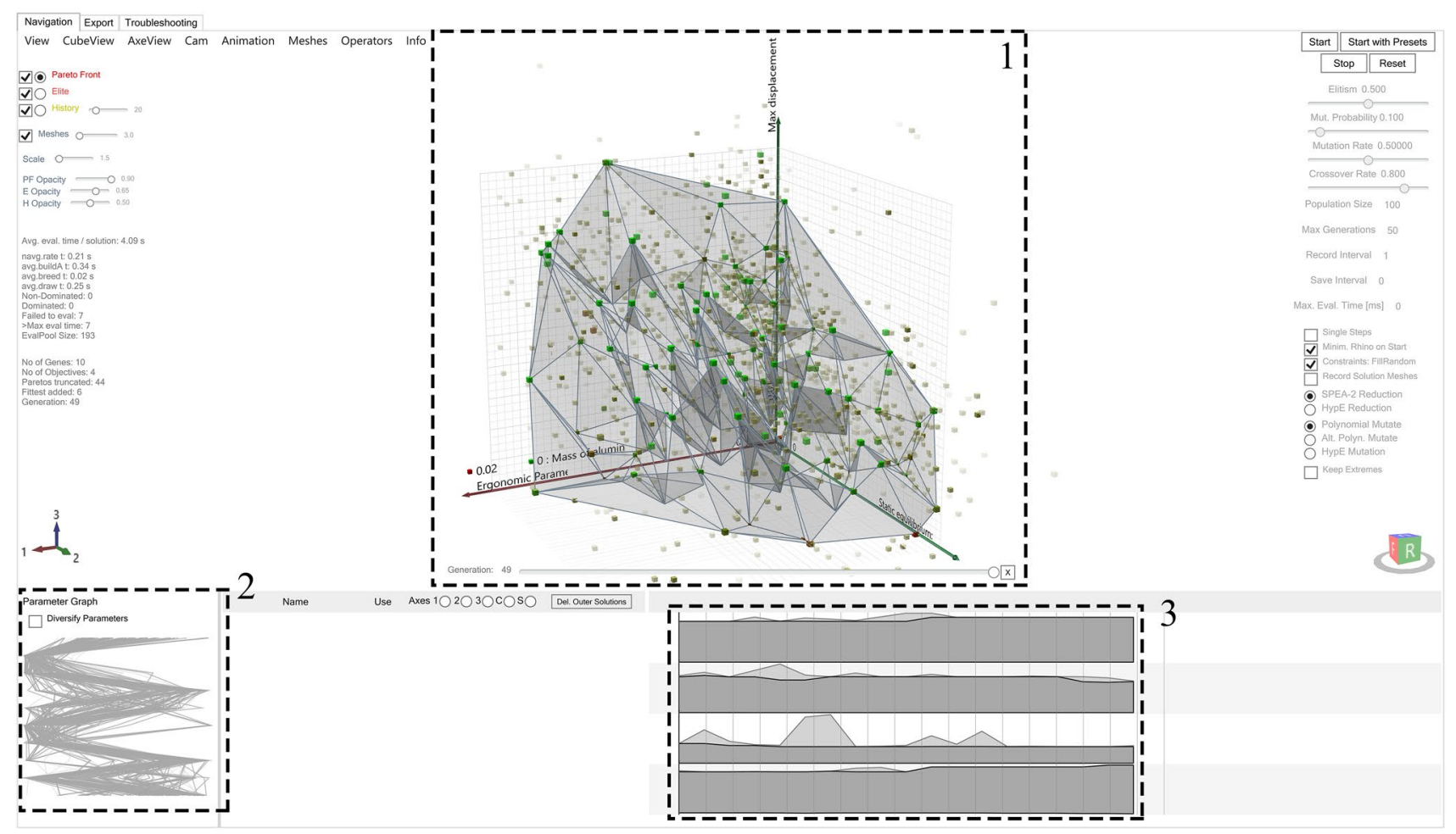

Fig. 7 Pareto optimal set 


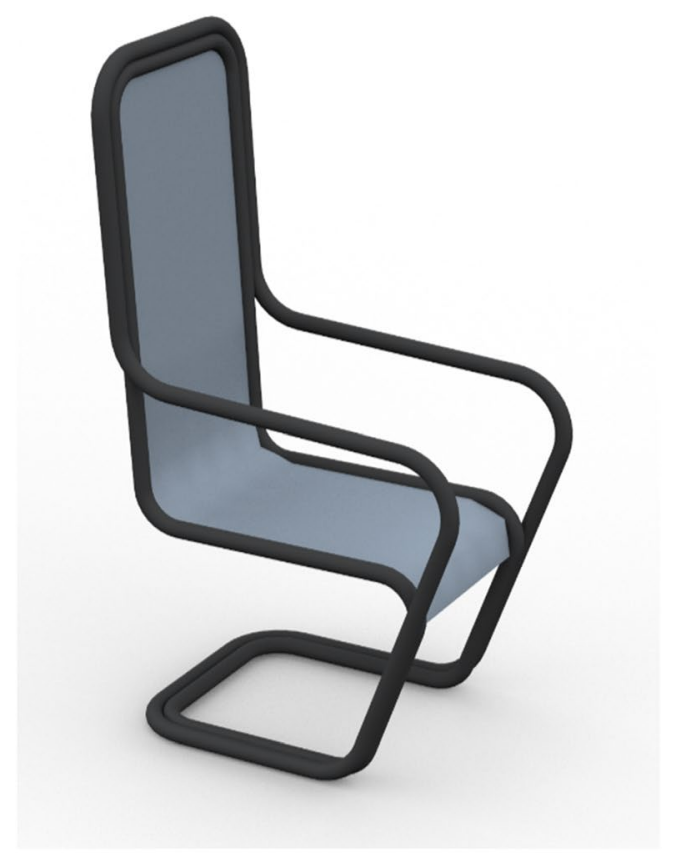

(a)

Fig. 8 Information layers: a rendered geometry; b structural model

has been generated by remap all the values assumed over the generations in the range between 0 and 1 , separately for each objective. The new data thus constituted will continue to represent performance visually and will make it possible to clearly read the performance trend in the various individuals. Figure 9a shows a radar chart where the performances of two distinct individuals are compared. The fitness values, of the all generations, have been remapped so that the minimum values are closer to the unit, since the purpose of the optimization is to minimize the objectives, while the higher fitness values, which identify lower performance, are close to 0 . In light of this, it is clear that the individual who corresponds to a greater area in the radar chart (red area) will perform better than the individual who corresponds to a minor underlying area (blue area). In the Fig. 9b are showed the corresponding design to the two different radar chart plotted in Fig. 9a. in the specific the chair on the left, corresponding to red area, and the chair on the right at the blue area. Figure 9c shows the Parallel coordinate plot chart in which each of the parallel axes corresponds to a different objective, also in this case the domains have been scaled between 0 and 1 . In this graph, the performance trend of all individuals present in the Pareto Optimal set is shown.

The case study, relating to the design of a chair, was chosen to test the proposed workflow. This workflow leads to the creation of an optimization tool, which, in addition to three-dimensional models, returns plots of explanatory graphs of the performance trend. The graphs and images

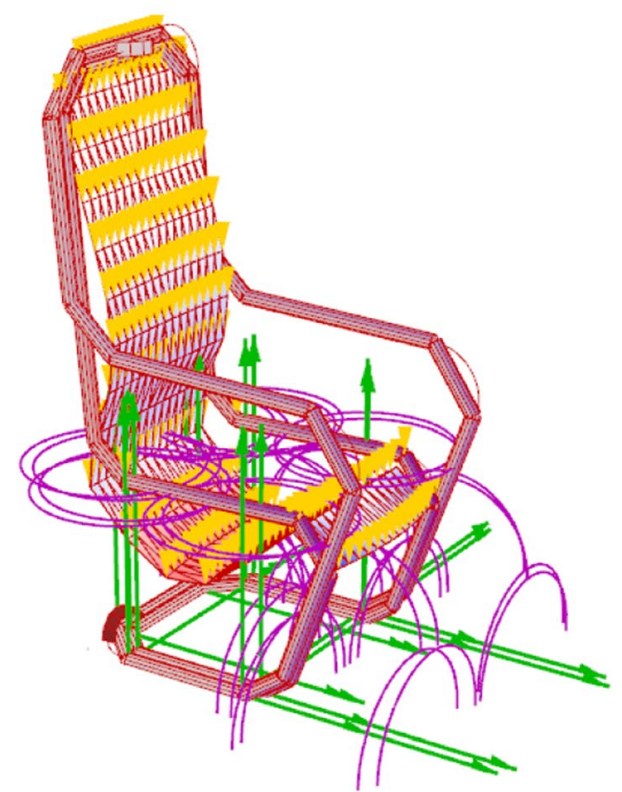

(b)

presented allow to evaluate the impact that this tool has on modeling both from a qualitative and quantitative point of view. The coupling of numerical data and graphic solutions, generated by the tool, allows the sorting of solutions based on the different parameters. This sorting process, in fact, can be carried out on the basis of the performance of the single target, or on the basis of the area underlying the radar chart, by ordering the best trade off solutions.

The results obtained from the sorting process are presented below. In Fig. 10a, b, c, d the upper quart percentile with respect to the four different optimization parameters are shown. For the generation of these results, reference was made to the dock of all 5000 solutions produced over the various generations. As can be seen, the extremes of the single objective, for example individuals who minimize the quantity of material, lead to economic solutions from the construction point of view but which do not satisfy with safety standards. However, taking into consideration the solutions that present only the minimization of deformations, individuals with high material consumption and negligible deformations characterized by structural oversizing are identified. Figure 10e represents and reports the global fittest solutions, that are the non-dominated solutions belonging to the Pareto optimal set. These solutions are easily identifiable through the radar plot chart because they correspond to the solutions that underlie the greater area. the extremism of the different objectives always corresponds to individuals that cannot be used for the development of real objects, 


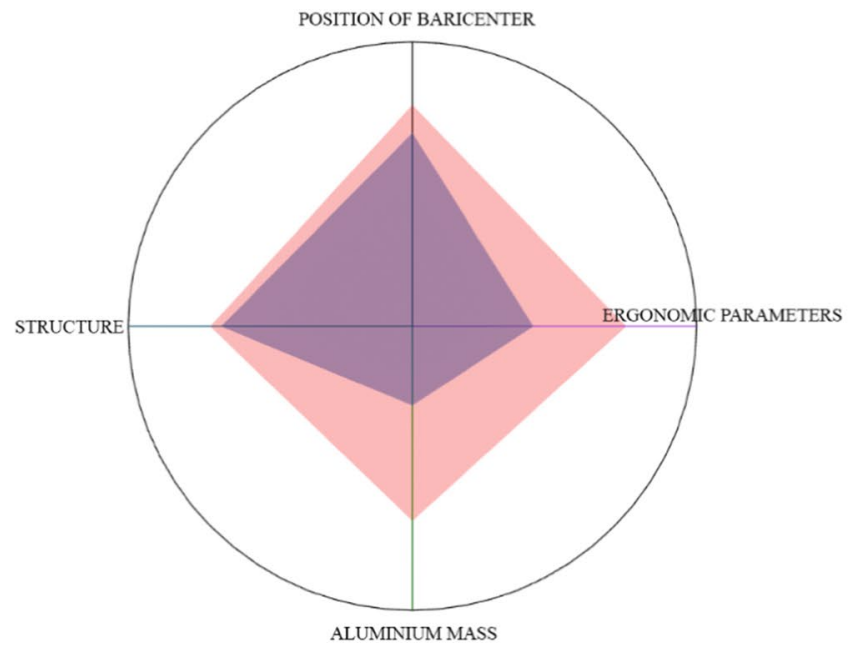

(a)

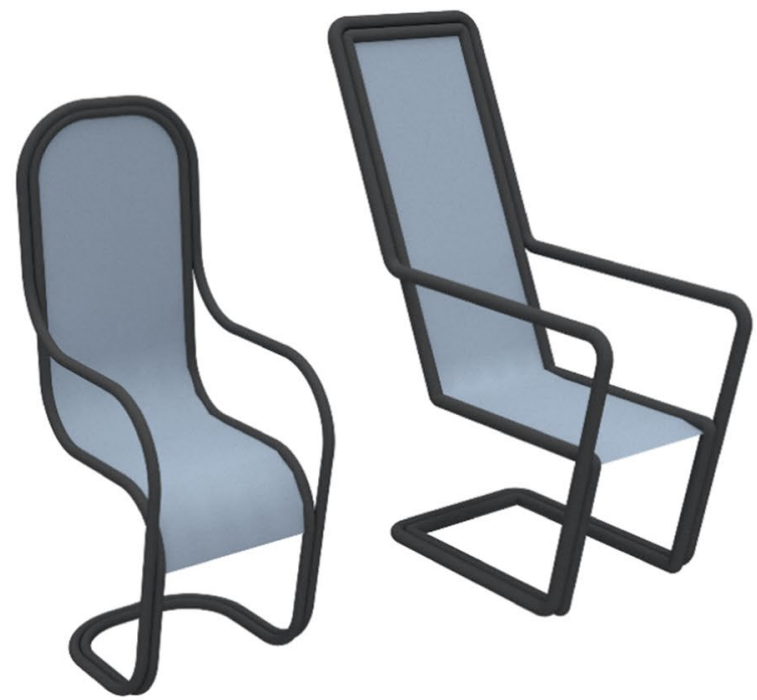

(b)

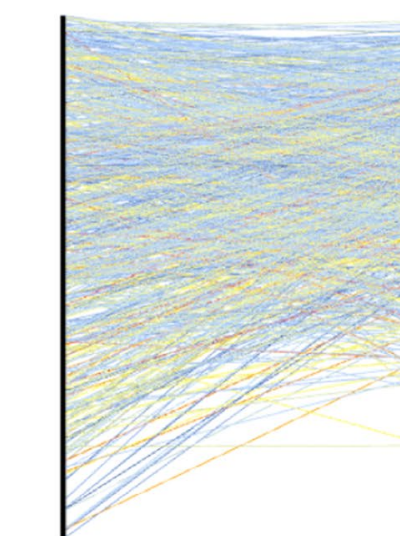

ERGONOMIC PARAMETERS

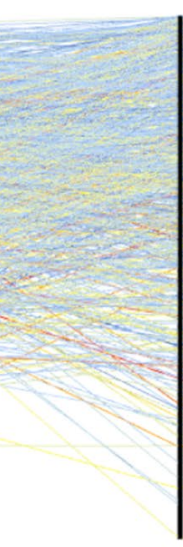

POSITION OF BARICENTER

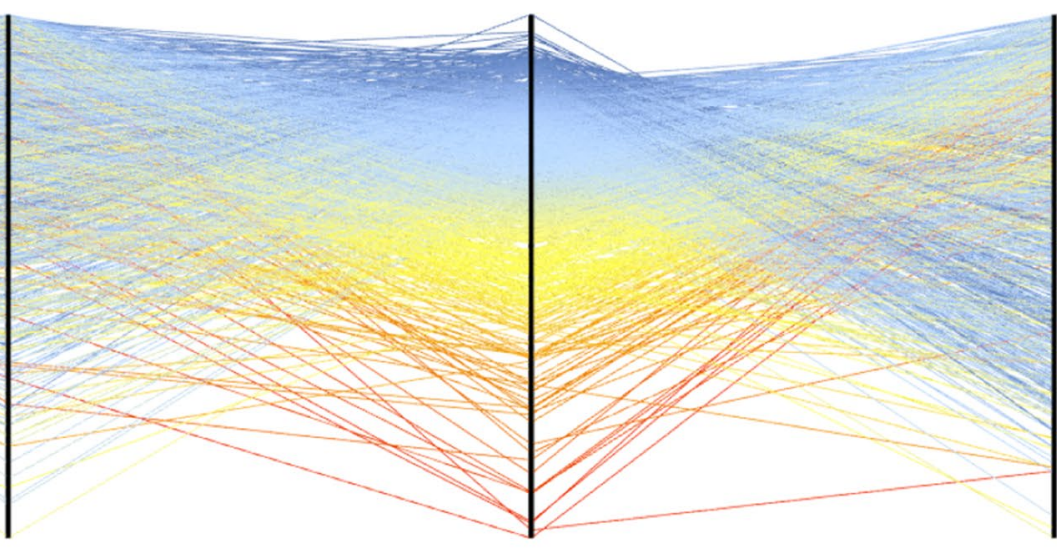

STRUCTURE

ALUMINIUM MASS

Fig. 9 Objective representation (a) Radar chart (b) design confronted (c) Parallel coordinate plot chart

while the tradeoff solutions that present less marked differences between one individual and another make them usable concretely.

\section{Conclusion and future work}

The principal aim of this paper is the creation of workflow, in order to help spread the optimization techniques within the manufacturing sectors in the process of industry 4.0. The proposed methods, exemplified through the case study, can be easily applied to any other definition of a three-dimensional model in grasshopper. The use of parametric modeling affects all production sectors; however, this process is not coupled with the implementation of Genetic Algorithms. This is due to the difficulty of interpreting the results and the slowness of the method. The workflow and the data obtained through the case study are a guide for designers in the sector who want to obtain and display optimized results, partially overcoming the limitations of the process. The use of this method for the chair object is nothing more than a first preliminary study of the application of genetic algorithms and generative systems to the prototipation and creation of design models. This has been useful to underline and illustrate the potential of the graphic tools applied by the new figures of computational designers who are insinuating into industry 4.0. 


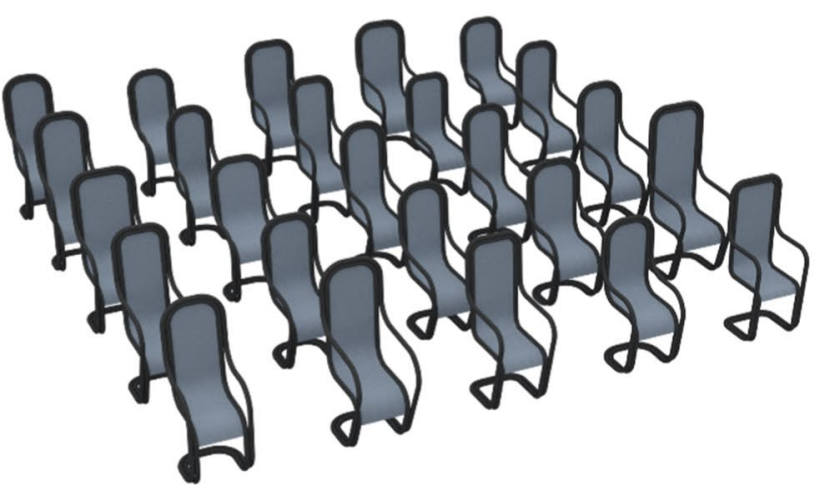

(a)

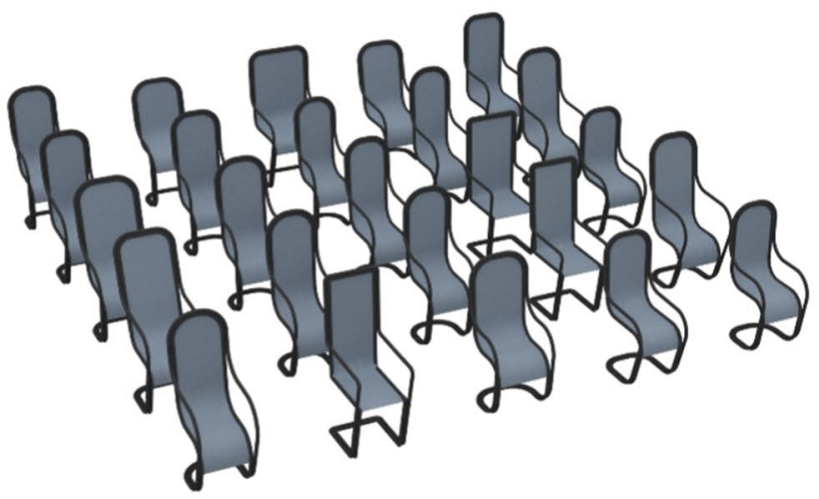

(c)

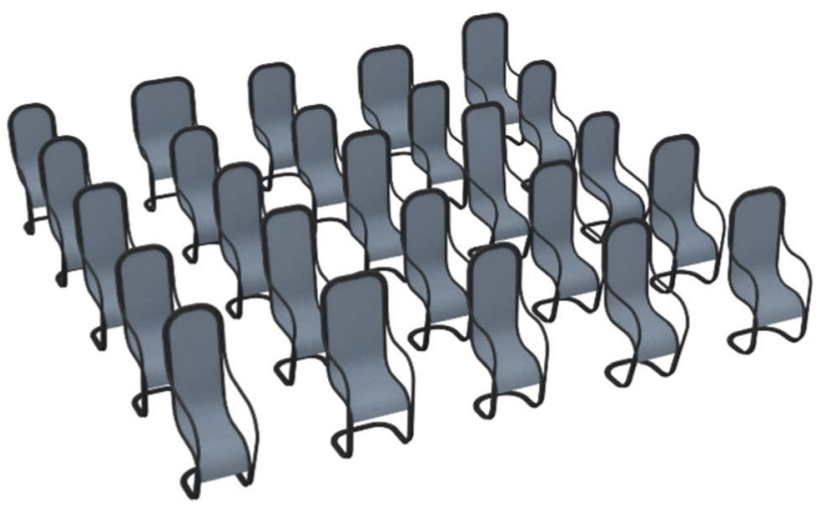

(b)

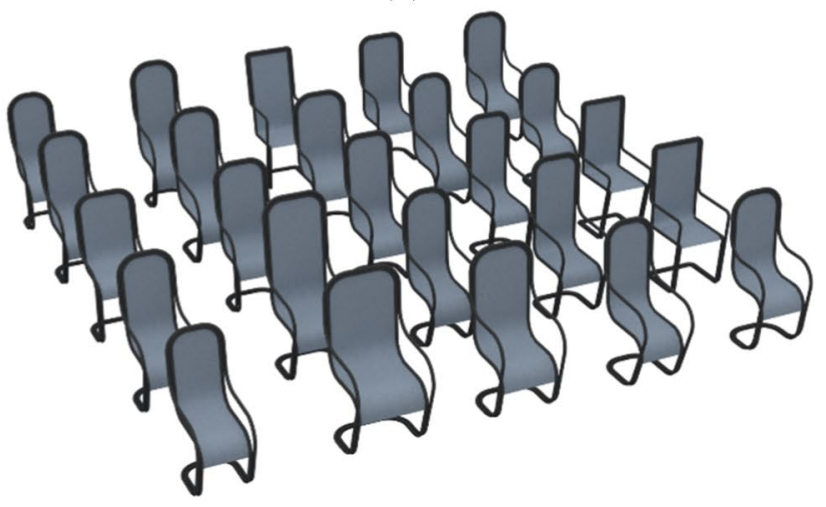

(d)

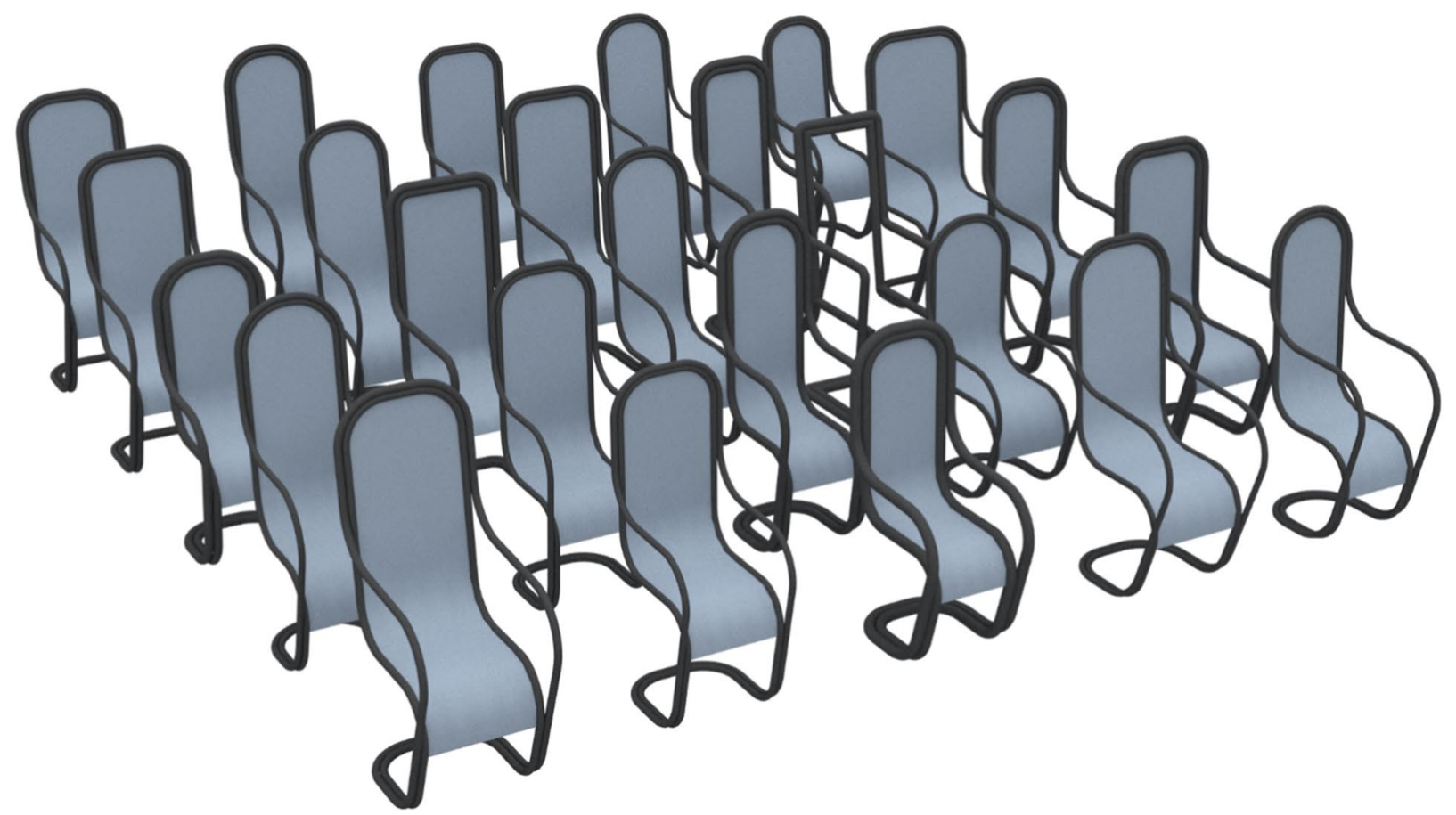

(e)

Fig. 10 Rapresentation of element in pareto optimal set 
Funding Open access funding provided by Università della Calabria within the CRUI-CARE Agreement

Open Access This article is licensed under a Creative Commons Attribution 4.0 International License, which permits use, sharing, adaptation, distribution and reproduction in any medium or format, as long as you give appropriate credit to the original author(s) and the source, provide a link to the Creative Commons licence, and indicate if changes were made. The images or other third party material in this article are included in the article's Creative Commons licence, unless indicated otherwise in a credit line to the material. If material is not included in the article's Creative Commons licence and your intended use is not permitted by statutory regulation or exceeds the permitted use, you will need to obtain permission directly from the copyright holder. To view a copy of this licence, visit http://creativecommons.org/licenses/by/4.0/.

\section{References}

1. 1335-1:2000, U. E. (s.d.). Sedie da lavoro per ufficio-Parte 1: dimensioni, determinazione delle dimensioni

2. 1335-2:2018. (s.d.). Sedie da lavoro per ufficio-Parte 2: requisiti di sicurezza

3. 1335-3:2009. (s.d.). Sedie da lavoro per ufficio—Parte 3: metodi di prova per la sicurezza

4. Abdechiri, M., Faez, K., Amindavar, H., Bilotta, E.: The chaotic dynamics of high-dimensional systems. Nonlinear Dyn. 87(4), 2597-2610 (2017)

5. Adamo, A., Bertacchini, P., Bilotta, E., Pantano, P., Tavernise, A.: Connecting art and science for education: learning through an advanced virtual theater with "talking heads". Leonardo 43(5), 442-448 (2010)

6. Aish, R., Woodbury, R.: Multy- level interaction in parametric desig. Smart Graphics 151-162 (2005)

7. Argyris, J.H., Papadrakakis, M., Apostolopoulou, C., Koutsourelakis, S.: The TRIC shell element: theoretical and numerical investigation. Comput. Methods Appl. Mech. Eng. 182(1-2), 217-245 (2000)

8. Ashour, Y., Kolarevic, B.: Optimizing creatively in multi-objective optimization. In: Proceedings of the SYMPOSIUM on Simulation for Architecture \& Urban Design (p. 128-135). Society for Computer Simulation International (2015)

9. Bader, J. M.: Hypervolume-based search for multiobjective optimization: theory and methods. Johannes Bader (2010)

10. Bader, J., Zitzler, E.: HypE: An algorithm for fast hypervolumebased many-objective optimization. Evolut. Comput. 45-76 (2011)

11. Bentley, P.J.: From Coffee Tables to Hospitals: Generic Evolutionary Design. Morgan kaufmann Publishers, San Francisco (1999)

12. Bentley, P. J.: Three Ways to Grow Designs: A Comparison of Embryogenies for an Evolutionary Design Problem. GECCO, 35-43 (1999)

13. Bertacchini, F., Tavernise, A.: Knowledge sharing for cultural heritage 2.0: pro-sumers in a digital agora. Int. J. Virtual Commun. Soc. Netw. (IJVCSN) 6(2), 24-36 (2014)

14. Bertacchini, F., Bilotta, E., Pantano, P.: Shopping with a robotic companion. Com-put. Hum. Behav. 77, 382-395 (2017)

15. Bertacchini, F., Bilotta, E., Pantano, P.: Discovery of Regular Domains in Large DNA Data Sets. In: Proceedings of the 8th ACM International Conference on Bioinformatics, Computational Biology, and Health Informatics, (pp. 744-749) (2017)

16. Bertacchini, F., Bilotta, E., Caldarola, F., Pantano, P.: The role of computer simulations in learning analytic mechanics towards chaos theory: a course experimentation. Int. J. Math. Educ. Sci. Technol. 50(1), 100-120 (2019)

17. Bertacchini, F., Bilotta, E., Caldarola, F., Pantano, P., Bustamante, L.: Emergence of linguistic-like structures in one-dimensional cellular automata. In: International Conference "Numerical Computations: Theory and Algorithms" (p. 090044). Pizzo Calabro,Italy: AIP Publishing (2016)

18. Bertacchini, F., Bilotta, E., Carini, M., Gabriele, L., Pantano, P., Tavernise, A.: Learning in the smart city: a virtual and augmented museum devoted to chaos theory. In: International Conference on Web-Based Learning (p. 261-270). Sinaia, Romania: Springer (2012)

19. Bertacchini, F., Bilotta, E., Gabriele, L., Olmedo Vizueta, D. E., Pantano, P., Rosa, F., Valentini, A.: An emotional learning environment for subjects with Autism Spectrum Disorder. In: International Conference on Interactive Collaborative Learning (ICL) (p. 653-659). Kazan, Russia: IEEE (2013)

20. Bertacchini, F., Bilotta, E., Gabriele, L., Pantano, P., Servidio, R.: Using Lego MindStorms in higher education: Cognitive strategies in programming a quadruped robot. In: Workshop proceedings 18th international conference on computers in education, ICCE (pp. 366-371). Putrajaya, Malaysia: Workshop Proceedings of the 18th International Conference on Computers in Education, ICCE 2010 (2010)

21. Bertacchini, F., Bilotta, E., Gabriele, L., Pantano, P., Tavernise, A.: Toward the use of Chua's circuit in education, art and interdisciplinary research: some imple-mentation and opportunities. Leonardo 46(5), 456-463 (2013)

22. Bertacchini, F., Rizzo, R., Bilotta, E., Pantano, P., Luca, A., Mazzuca, A., LopezInitiative, A.A.F.: Mid-sagittal plane detection for advanced physiological measurements in brain scans. Physiol. Meas. 40(11), 115009 (2019)

23. Bilotta, E., Pantano, P.: Artificial micro-worlds part II: cellular automata growth dynamics. Int. J. Bifurcat. Chaos 21(3), 619-645 (2011)

24. Bilotta, E., Bossio, E., Pantano, P.: Chaos at School: Chua's circuit for students in junior and senior High School. Int. J. Bifurcat. Chaos 20(1), 1-28 (2010)

25. Bilotta, E., Di Blasi, G., Stranges, F., Pantano, P.: A gallery of Chua attractors part VI. Int. J. Bifurcat. Chaos 17(6), 18011910 (2007)

26. Bilotta, E., Di Blasi, G., Stranges, F., Pantano, P.: A gallery of Chua attractors: part IV. Int. J. Bifurcat. Chaos 17(4), 10171077 (2007)

27. Bilotta, E., Pantano, P., Stranges, F.: Computer graphics meets chaos and hyperchaos: some key problems. Comput. Graph. 30(3), 359-367 (2006)

28. Bilotta, E., Pantano, P., Vena, S.: Artificial micro-worlds part I: a new approach for studying life-like phenomena. Int. J. Bifurcat. Chaos 21(2), 373-398 (2011)

29. Bilotta, E., Pantano, P., Vena, S.: Speeding up cellular neural network processing ability by embodying memristors. IEEE Trans. Neural Netw. Learn. Syst. 28(5): 1228-1232). IEEE (2016)

30. Bilotta, E., Pantano, P., Bertacchini, F., Gabriele, L., Longo, G., Mazzeo, V., Vena, S.: ImaginationTOOLS (TM)-A 3D environment for learning and playing music. In: Eurographics Italian Chapter Conference (pp. 139-144). Trento: Raffaele De Amicis and Giuseppe Conti (Editors) (2007)

31. Bilotta, E., Stranges, F., Pantano, P.: A gallery of Chua attractors: part III. Int. J. Bifurcat. Chaos 17(3), 657-734 (2007)

32. Bradner, E., Iorio, F., Davis, M.: Parameters tell the design story: ideation and abstraction in design optimization. In: Proceedings of the symposium on simulation for architecture \& urban design, 26-33 (2014) 
33. Brintrup, A. M., Ramsden, J., Takagi, H., Tiwari, A.: Ergonomic chair design by fusing qualitative and quantitative criteria using interactive genetic algorithms. IEEE Transactions on Evolutionary Computation, (p. 343-354) (2008)

34. Cho, S.B.: Towards creative evolutionary systems with interactive genetic algorithm. Appl. Intell. 16(2), 129-138 (2002)

35. Deb, K.: Multi-objective optimization using evolutionary algorithms. Chichester, U.K.: S. Ross and R. Weber, Eds (2001)

36. Demarco, F., Bertacchini, F., Scuro, C., Bilotta, E., Pantano, P.: Algorithms for Jewelry Industry 4.0. Numerical Computations: Theory and Algorithms NUMTA 2019 (pp. 425-436). Le Castella, Crotone, Italy: Springer (2019)

37. Frazer, J.: An Evolutionary Architecture. Architectural Association, London (1995)

38. Gabriele, L., Bertacchini, F., Tavernise, A., Vaca-Cárdenas, L., Pantano, P., Bilotta, E.: Lesson planning by computational thinking skills in italian pre-service teachers. Inform. Educ. 18(1), 69-104 (2019)

39. Gabriele, L., Marocco, D., Bertacchini, F., Pantano, P., Bilotta, E.: An educational robotics lab to investigate cognitive strategies and to foster learning in an arts and humanities course degree. Int. J. Online Biomed. Eng. 13(04), 7-19 (2017)

40. Gabriele, L., Tavernise, A., Bertacchini, F.: Active learning in a robotics laboratory with university students. Cutting-Edge Technologies in Higher Education (6)(Part C), 315-339 (2012)

41. Giglio, S., Bertacchini, F., Bilotta, E., Pantano, P.: Using social media to identify tourism attractiveness in six Italian cities. Tourism management, 306-312 (2019)

42. Giglio, S., Bertacchini, F., Bilotta, E., Pantano, P.: Machine learning and points of interest: typical tourist Italian cities. Current Issues in Tourism, 1-13 (2019)

43. Harding, J. J.: Thinking topologically at early stage parametric design (2012)

44. Hensen, J. L., Lamberts, R.: Building performance simulation for design and operation. Routledge (2012)

45. Hillberg, C., Andersson, V.: Evolutionary optimization of steel truss footbridges-A study of parametric design and optimization in Karamba 3D, Galapagos and Octopus for different truss geometries (2018)

46. Holzer, D., Hough, R., Burry, M.: Parametric design and structural optimisation for early design exploration. Int. J. Arch. Comput. 625-643 (2007)

47. Kolarevic, B., Malkawi, A.: Eformative Architecture: Beyond Instrumentality. Routledge (2005)

48. Lili, W., Tao, S., Zheng, Y., Yixiong, L.:I The parametric design of office chairs based on ergonomics. In: International Conference on Computing, Control and Industrial Engineering, (pp. 355-360) (2010)

49. Lombardo, M., Barresi, R., Bilotta, E., Gargano, F., Pantano, P., Sammartino, M.: Demyelination patterns in a mathematical model of multiple sclerosis. J. Math. Biol. 75(2), 373-417 (2017)

50. Piscioneri, A., Morelli, S., Mele, M., Canonaco, M., Bilotta, E., Pantano, P., Drioli, E., De Bartolo, L.: Neuroprotective effect of human mesenchymal stem cells in a compartmentalized neuronal membrane system. Acta Biomater. 24, 297-308 (2015)

51. Preisinger, C.: Karamba3D. Version 1(3), 2 (2018)

52. Reed, K., Gillies, D.: ChairMaker-A Parametric Chair Modelling Program. Imperial College London, London (2016)

53. Rubin, H., Schneider, K. J.: Baustatik-Theorie I. und II. Ordnung: Werner (1996)

54. Scuro, C., Lamonaca, F., Codispoti, R., Carnì, D.L., Olivito, R.S.: Experimental and numerical analysis on masonry arch built with fictile tubules bricks. Measurement 130, 246-254 (2018)

55. Scuro, C., Tiberti, S., Codispoti, R., Milani, G., Olivito, R.S.: Fictile tubules: a traditional Mediterranean construction technique for masonry vaulted systems. Constr. Build. Mater. 193, 84-96 (2018)

56. Singh, V., Gu, N.: Towards an integrated generative design framework. Esign Studies, 185-207 (2012)

57. Vaca-Cárdenas, L. A., Bertacchini, F., Tavernise, A., Gabriele, L., Valenti, A., Olmedo, D. E., Bilotta, E.: Coding with Scratch: the design of an educational setting for Elementary pre-service teachers. In: 2015 International Conference on Interactive Collaborative Learning (ICL) (pp. 1171-1177). IEEE (2015)

58. Vierlinger, R.: Octopus. Bollinger+ Grohmann Engineers (2014)

59. Vierlinger, R., Hofmann, A.: A Framework for flexible search and optimization in parametric design. In: Proceedings of the Design Modeling Symposium. Berlin (2013)

60. Viola, E., Federici, L., Nobile, L.: Detection of crack location using cracked beam element method for structural analysis. Theor. Appl. Fract. Mech. 36, 23-35 (2001)

61. Zitzler, E., Laumanns, M., Thiele, L.: SPEA2: Improving the strength Pareto evolutionary algorithm. TIK-report, 103 (2001)

Publisher's Note Springer Nature remains neutral with regard to jurisdictional claims in published maps and institutional affiliations. 